\title{
Adaptive primary user's signal detection algorithm based on matrix accumulation estimation for cognitive radio
}

\author{
Hongjun Wang ${ }^{1,2^{*}}$, Guangguo Bi ${ }^{1 *}$, Zifa Zhong ${ }^{2}$, Shuo Zhang $^{2}$ and Zaichen Zhang ${ }^{1}$
}

\begin{abstract}
In this article, a new adaptive primary user's signal detection algorithm for cognitive radio, which integrates frequency-domain block adaptive filtering with a matrix accumulation estimation, is presented with detailed description' of the concept and process of the algorithm. Then, the performance of the algorithm is analyzed and compared by simulation according to the practical requirement of cognitive radio. The result shows that the proposed detection algorithm can detect weak signals quickly and effectively with higher detection probability.
\end{abstract}

Keywords: cognitive radio, signal detection, block-FFT adaptive filter, matrix accumulation estimation

\section{Introduction}

The cognitive radio [1-5] can sense and recognize the changes of the electromagnetic environment and borrow a spectrum-hole to transmit information in real-time manner without bringing about any interference to primary users and other users around. It must release the "borrowed" channels and switch to the next spectrumhole as soon as primary users appear. The cognition must run through the whole communication process. The chief task of cognitive radio is to scout and determine the spectrum-hole, that is, cognitive radio should have the ability of detecting weak primary user signals from strong background noise [6-8].

Since the digital filters was presented, the adaptive filters technology especially the adaptive least mean square (LMS) filters have been applied widely in scientific and technological domains such as communication systems and signal processing systems. Among the adaptive filters, the adaptive block LMS filters [9-11] is an more efficient and faster scheme to detect signals in background noise. The latest research findings shows the adaptive block filters provide almost the same meansquare-error as the recursive least squares adaptive filter and possesses a superior convergence rate than the

\footnotetext{
* Correspondence: hongjun-wang@163.com; bigg@seu.edu.cn 'National Mobile Communications Research Laboratory, Southeast University, Nanjing 210096, China

Full list of author information is available at the end of the article
}

conventional LMS and the normalized LMS filters $[12,13]$. The block LMS adaptive filter processes data on a block-by-block basis, as opposed to a sample-by-sample basis. Chia-Chang $\mathrm{Hu}$ and his collaborators presented an adaptive block DFT-LMS filter to detect users' signals in the impulse radio ultra-wideband (IRUWB) systems in [12]. Based on the LMS method, Atrouz adopted the partial adaptive scheme and the block processing to complete the jammers cancellation in paper [13]. From the results of the two papers mentioned above, both algorithms will be difficult to accomplish the target under $-1.2 \mathrm{~dB}$ SINR.

Our application scene is GSM mobile system, the aim of the research project is to detect the GMSK mobile signal, find out the idle time slot and borrow it to communicate among the cognitive transceivers. The SNR is about $-5 \mathrm{~dB}$ by actual measuring because of the adjacent channel interferences, the co-channel interferences and the SNR walls effect. It's obvious that the algorithms mentioned above will be difficult to detect the weak primary user's signal from the strong background noise, and the probability of these detections will be low in low SNR or boundary detection. How to improve the detection probability and detect the primary user's signal is our chief task.

Because of its low computational complexity and good convergence property, the block-FFT adaptive filtering algorithm is adopted by our new adaptive detection 
algorithm of the primary user's signal, which is proposed on basis of the matrix accumulation estimation to overcome further the shortcoming of the above-mentioned algorithms.

\section{Adaptive detection algorithm of the primary user's signal}

The application scene is the mobile wireless environment. As known to all, the idle rate of time-slots is up to $60 \%$ in GSM mobile system at non-dense district or non-dense time. How to use the idle time-slots sufficiently is a interesting topic especially to spectrum resource deficiency nowadays. Our objective lies to detect and use the idle time-slots by cognitive technology. The cognitive radio equipments have multiplex signal detection channels in our design as Figure 1.

In Figure 1, RF Rx. represents the RF receiver channel, which is designed to detect the weak primary user's signal with the A/D module and the digital signal processor. And RF Trans. stands for the RF transmitter channel, which is designed to communicate with other cognitive radio equipments. Each detection channel of the cognitive radio equipments begins to detect the weak primary user's signal in the frequencybandwidth assigned by the digital signal processor after startup. Then the idle spectrum is collected as the spectrum-holes. According to stipulation regulation, the cognitive radio equipments may communication between spectrum-holes detected. The detection processing runs through the whole communication process. Being confined to the length of the paper, we will only discuss the weak primary user's signal detection algorithm in detail in the paper.

In most cases, the rate of fading processing is slower than the symbol rate of the signal in GSM mobile system, so the Rice amplitude fading model is adopted in algorithm simulation in view of the existence of the multipath signals. The binary hypothesis is used to test the algorithm as follows.

$$
x(t)=\left\{\begin{array}{cc}
n(t), & H_{0} \\
h(t) \otimes s(t)+n(t), & H_{1}
\end{array}\right.
$$

where, $x(t)$ is the signal received by the cognitive users, and $s(t)$ is the signal sent by the primary users. $h$ $(t)$ is the channel fading coefficient, its probability density function is.

$p(r)=\left\{\begin{array}{ll}\frac{r}{\sigma^{2}} \exp \left(-\frac{r}{2 \sigma^{2}}\right) & (0 \leq r \leq \infty) \\ 0 & r<0\end{array} \cdot n(t)\right.$ is the noise. $H_{0}$ indicates the absence of the primary users, and $H_{1}$ indicates the existence of the primary users.

Figure 2 shows the process of our new adaptive detection algorithm to the primary user's signal which is performed in digital signal processor, where BFFTAA is the abbreviation of the Block-FFT adaptive filter algorithm. The MAE represents the matrix accumulation estimation, The new algorithm adopts the speediness and degrading-noise advantage of the BFFTAA and the MAE, and is named BFFTAA-MAE algorithm for brevity.

\subsection{The block-FFT adaptive filtering algorithm}

$x(n)$ is the discrete time data of $x(t)$, and is divided into $M$ segments in BFFTAA-MAE algorithm, and each segment has $N$ samples. $N$ is the number of the filter tap coefficients. In order to express our viewpoint, the iteration equation of the $k$-th block data is given as following.

$$
\mathbf{W}(k+1)=\mathbf{W}(k)+u \sum_{i=0}^{N-1} e(k N+i) \mathbf{X}(k N+i)=\mathbf{W}(k)-\frac{1}{2} u \nabla(k)
$$

and

$$
y(k N+i)=\mathbf{W}^{T}(k) \mathbf{X}(k N+i) \quad i=0,1, \cdots, N-1
$$

where $k$ is the index of the $k$-th block data, $y(k N+i)$ is the time-domain output of the LMS filters at the $k$-th

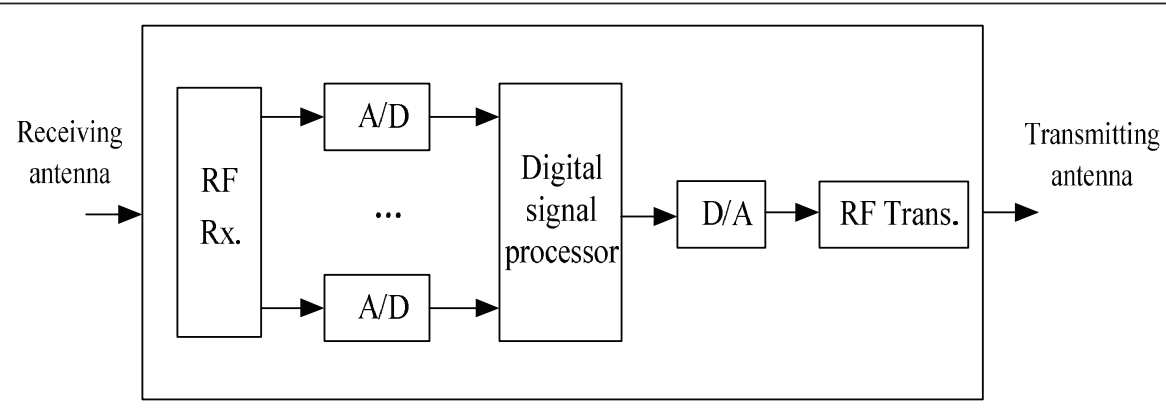

Figure 1 The principle block diagram of the cognitive radio equipment. 


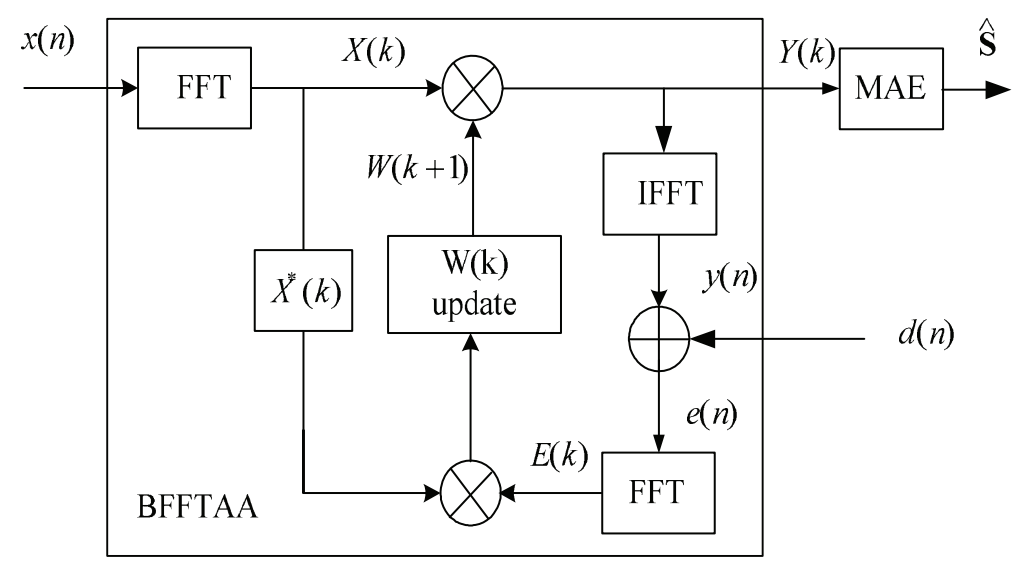

Figure 2 The process of adaptive spectrum-hole detection algorithm.

block data, and $\mathbf{w}(k)$ is the weight coefficients vector of the filters corresponding to the $k$-th block data.

$$
\mathbf{W}(k)=\left[\begin{array}{llll}
w_{0}(k) & w_{1}(k) & \cdots & w_{N-1}(k)
\end{array}\right]^{T}
$$

and $\mathbf{X}(n)$ in Equation (2) is the up-to-date $N$ samples of the filter input at the $n$-th instant, which is also regarded as the output of the delay line with $N$ taps.

$$
\mathbf{X}(n)=\left[\begin{array}{llll}
x(n) & x(n-1) \quad \cdots \quad x(n-N+1)
\end{array}\right]^{T}
$$

$e(n)$ is the error sequence in Equation (2), which equals the difference between the expected response $d$ (n) and the output of the filter $y(n)$ as Equation (6), and $u$ is the update step of the weight coefficient.

$$
e(n)=d(n)-\gamma(n)
$$

Because of the GSM mobile application scene, the expected response $d(n)$ may be regarded deterministic. The GMSK signal is adopted as the expected primary user's signal $d(n)$ in subsequent simulation.

On the strength of derivation above, the BFFTAA algorithm is presented hither. In order to reduce the computational complexity of BFFTAA, the overlapped reservation algorithm is adopted in frequency-domain with $50 \%$ data overlapped reservation. The overlapped reservation algorithm also increases the efficiency of the primary user's signal detection algorithm afterwards. The discrete FFT is performed with $N$ samples per segment, meanwhile the weight coefficients are replaced according to the accumulation result of the error sequence.

The concrete process is to insert $N$ zeros at the tail of $\mathbf{w}(k)$ firstly, then to perform $2 N$-FFT to obtain the weight coefficients vector of the frequency-domain filtering as follows.

$$
\left[\mathbf{W}^{k}(f)\right]^{T}=F F T\left[\mathbf{W}^{T}(k) \quad 0 \quad \cdots \quad 0\right]
$$

where, $\mathbf{w}^{k}(f)$ is a $2 N \times 1$ vector of the $k$-th block data, and the length of vector in frequency-domain is twice than the length of vector in time-domain.

Then $\mathbf{X}^{k}(f)$ is obtained firstly by performing $2 N$-FFT to $(k-1)$-th data block and $k$-th data block, and diagonalizing the result of FFT as Equation (8)

$$
\mathbf{X}^{k}(f)=\operatorname{diag}\{F F T[x(k N-N) \quad \cdots \quad x(k N-1) \quad x(k N) \quad \cdots \quad x(k N+N-1)]\}
$$

Finally the result of the BFFTAA algorithm is the last $N$ items of the Equation (9):

$$
\mathbf{Y}^{k}(f)=\left[\mathbf{Y}_{0}^{k}(f), \mathbf{Y}_{1}^{k}(f), \cdots, \mathbf{Y}_{N-1}^{k}(f)\right]^{T}=\underbrace{\left[\mathbf{X}^{k}(f) \mathbf{W}^{k}(f)\right]}_{\text {The last Nitems }}
$$

\subsection{Matrix accumulation estimation}

Although the BFFTAA algorithm reduces the computation complexity, it does not improve the detection performance. In order to improve the detection performance, the matrix accumulation estimation (MAE) is integrated with the BFFTAA as a whole.

$\mathbf{Y}^{k}(f)$ firstly is conjugated, then a $N \times N$ Hankel matrix is reshaped as follows.

$$
\mathbf{B}^{k}(f)=\left[\begin{array}{cccl}
\mathrm{Y}_{0}^{k}(f) & \mathrm{Y}_{1}^{k}(f) & \cdots & \mathrm{Y}_{N-1}^{k}(f) \\
\mathrm{Y}_{1}^{k}(f) & \mathrm{Y}_{2}^{k}(f) & \cdots & \mathrm{Y}_{0}^{k}(f) \\
\cdot & \cdot & & \cdot \\
\cdot & \cdot & & \cdot \\
\mathrm{Y}_{N-1}^{k}(f) & \mathrm{Y}_{0}^{k}(f) & \cdots & \mathrm{Y}_{N-2}^{k}(f)
\end{array}\right]
$$

Then $\mathbf{C}^{k}(f)$ is obtained by a matrix accumulation calculation as Equation (11)

$$
\mathbf{C}^{k}(f)=\left[\mathbf{Y}^{k}(f)\right]^{T}\left[\mathbf{Y}^{k}(f)\right]
$$


After completing the above processing, we define a matrix called $\mathbf{S}^{k}(f)$ :

$$
\mathbf{S}^{k}(f)=\left[\begin{array}{cccl}
S_{0,0}^{k}(f) & S_{0,1}^{k}(f) & \cdots & S_{0, N-1}^{k}(f) \\
S_{1,0}^{k}(f) & S_{1,1}^{k}(f) & \cdots & S_{1, N-1}^{k}(f) \\
\cdot & \cdot & & \cdot \\
\cdot & \cdot & & \cdot \\
S_{N-1,0}^{k}(f) & S_{N-1,1}^{k}(f) & \cdots & \mathrm{Y}_{N-1, N-1}^{k}(f)
\end{array}\right]
$$

The matrix multiplication estimation is performed as Equation (13).

$$
\begin{aligned}
\mathbf{S}^{k}(f) & =\mathbf{C}^{k}(f) \times \mathbf{B}^{k}(f) \\
& =\left\{\left[\mathbf{Y}^{k}(f)\right]^{T}\left[\mathbf{Y}^{k}(f)\right]\right\} \times\left[\begin{array}{cccc}
\mathrm{Y}_{0}^{k}(f) & \mathrm{Y}_{1}^{k}(f) & \cdots & \mathrm{Y}_{N-1}^{k}(f) \\
\mathrm{Y}_{1}^{k}(f) & \mathrm{Y}_{2}^{k}(f) & \cdots & \mathrm{Y}_{0}^{k}(f) \\
\cdot & \cdot & & \cdot \\
\cdot & \cdot & & \cdot \\
\mathrm{Y}_{N-1}^{k}(f) & \mathrm{Y}_{0}^{k}(f) & \cdots & \mathrm{Y}_{N-2}^{k}(f)
\end{array}\right]
\end{aligned}
$$

Equation (13) has the similar expression to the higher accumulation statistic estimation [14].

$$
\mathbf{S}^{k}(f) \sim Y^{k}\left(\lambda_{1}\right) \cdot Y^{k}\left(\lambda_{2}\right) \cdot\left[Y^{k}\left(\lambda_{1}+\lambda_{2}\right)\right]^{*}
$$

The essence of the Equation (13) is to calculate a matrix about third-order accumulation statistic. The third-order matrix product is computed in the algorithm. The cumulant is a valid method to suppress the noise $[15,14]$, so the noise will be further suppressed in the algorithm, and the SNR will be raised correspondingly. The subsequent simulation will verify our viewpoint.

Then the accumulated product $\hat{\mathbf{S}}$ is acquired by frequency-domain smoothing to $\mathbf{S}^{k}(f)$. Its essence is to execute two dimensional convolution to $\mathbf{S}^{k}(f)$ with $\mathbf{F}_{(m, n)}$. The elements of $\mathbf{F}_{(m, n)}$ will be obtained as Equation (15).

$$
F_{(m, n)}=\frac{\sqrt{3}}{\pi^{3}}\left[1-\frac{m^{2}+n^{2}+m n}{N^{2}}\right]
$$

where $\mathbf{F}_{(m, n)}$ is a Rao-Gabr window [15], and $m^{2}+n^{2}+m n \leq \frac{\mathbf{F}_{(m, n)}^{2}}{(N / 2)^{2}}(m, n) \in G, G$ is the sets of sampling points.

Finally, $\hat{S}$, which is the estimated value of the accumulated product $\hat{\mathbf{S}}$, is derived by operation of Mod as Equation (16).

$$
\hat{S}=\frac{1}{M} \sum_{k=1}^{M}\left(\frac{1}{N} \sum_{i=0}^{N-1}|\hat{\mathbf{S}}|\right)
$$

\subsection{The weight-coefficient update}

According to the above result, the weight-coefficient update and the primary user's signal detection are calculated in the meantime in the subsequent process.
FFT of the error sequence is transformed as Equation (17).

$$
\mathbf{E}^{k}(f)=\operatorname{FFT}[0 \quad \cdots \quad 0 \quad(d(k N)-\gamma(k N)) \quad \cdots \quad(d(k N+N-1)-\gamma(k N+N-1))]
$$

Then, the gradient is obtained by extracting the $N$ items of Equation(18).

$$
\nabla(k)=-2 \mathrm{FFT}^{-1}\left[\mathrm{X}^{*}(k) \mathrm{E}(k)\right]
$$

Finally, the weight-coefficient update formula is gained as follows:

$$
\mathbf{W}^{k}(f+1)=\mathbf{W}^{k}(f)+\frac{1}{2} u \mathrm{FFT}\left[\begin{array}{l}
\nabla(k) \\
0 \\
\vdots \\
0
\end{array}\right]
$$

Then $\mathbf{S}^{k}(f)$ will be updated in the wake of the iteration and renewal to the parameters and data mentioned above.

\subsection{Detection determination of the primary user's signal}

The spectral components have a homogeneous distribution in frequency domain after denoising with the adaptive detection algorithm. Taking into account the protection band between authorized frequency-bands, the noise level may be estimated in upper protection band or lower protection band beyond the frequencyband to be detected. In accordance with the Equation (16), $\hat{S}_{P B}$ is calculated by the BFFTAA-MAE algorithm to the data sampled in the protection band.

Then $\hat{S}_{I B}$ is calculated with the data sampled in-band according to the Equation (16). $\hat{S}_{P B}$ is defined as the determination threshold. While, $\hat{S}_{I B} \geq \hat{S}_{P B}$ the $H_{1}$ hypothesis is true, and the primary users is supposed to exist. Otherwise $H_{0}$ is accepted, and the processed data is decided as noise. The empirical value of $\hat{S}_{I B}$ is often $0.5 \mathrm{~dB}$ greater than $\hat{S}_{P B}$ in order to constrain noise better, that's to say, $\hat{S}_{I B} \geq \hat{S}_{P B}+0.5$. But the adjusted value cannot be set too great in case the process loses the superabundance information of the primary users.

According to the determination, the cognitive equipments will detect the primary user's signal, and may communicate through the idle channels.

\section{Simulation and performance analysis of the algorithm}

Both the adaptive filtering and the multiple matrix accumulative algorithm are efficient means to overcome the strong background noise. The major advantage of the algorithm lies in its constant false alarm 


$$
\begin{array}{|l|l|l|l|l|l|}
\hline 3 \text { trail bits } & 57 \text { information bits } & 00101101110111100010110111 & 57 \text { information bits } & 3 \text { trail bits } & 8.25 \text { gurad bits } \\
\hline
\end{array}
$$

Figure 3 The baseband data of the signal.

rate. The Monte Carlo is adopted in simulation. The signals of the primary users are supposed to be the GMSK baseband modulating signal of the GSM mobile system. There are eight time-slots with different normal train sequence in a normal frame, and each timeslot has 156.25 bits. The eight normal train sequences of the GSM mobile system are applied to format the baseband data of the primary user according the GSM standard. For example, the data of the primary user, which is supposed to be detected in our algorithm, is given as Figure 3. Where the train sequence of the primary user is 00101101110111100010110111 , and the 57 information bits, 3 trail bits, and 8.25 guard bits are generated by random generator. The $d(n)$ is certainly obtained with the same train sequence. The 26 bits of $d(n)$ is used to track and converge the primary signal. In simulation, other seven bursts are continuous to transmit the data according to the different normal train sequences except the train sequence mentioned above. Meanwhile the data of the primary user, which will be detected, is transmitted now and then. That's to say, the primary user is exit while the data of the primary user is transmitted, otherwise the primary user is absent.

In order to ensure get the whole data of eight timeslots and better simulation result, the data with 8,192 points is adapted in the simulation. Meanwhile in order to reduce the computational complexity and raise the computational speed, the data is divided into 32 blocks for overlapped reservation, and each segment has 64 points. It is well known that the computation burden will obviously increase if the length of the Rao-Gabr window is selected too long, and the effect will be bad if the length of the Rao-Gabr window is selected too short. It is acceptable that the length of the Rao-Gabr window is $(7,7)$ according to the result of simulation. The range of $\mu$ is from 0 to 1 . The constringency rate will be reduced if $\mu$ is set too big. It can meet the real-time of the algorithm that $\mu$ is set as 0.01 according to the result of simulation. SNR is set from $-10 \mathrm{~dB}$ to $10 \mathrm{~dB}$, and the white noise is adapted in the simulation. Without loss of generality, the Rice amplitude fading model is applied in simulation.

In order to illustrate the superiority of the BFFTAAMAE detection, the block DFT filters detection [9] and the block LMS detection [10] are compared by computer simulation under the same condition at the same time. Being confined to the length of the paper, we only compared such three algorithms. We find that $-5.8 \mathrm{~dB}$ is the performance watershed of these algorithms in the simulation, so $-5.8 \mathrm{~dB}$ SNR will be applied while we analyze the performance of these algorithms.

Firstly, we get the spectral-peak figures of three algorithms mentioned above at $-5.8 \mathrm{~dB}$ SNR. CSI means comparative signal intensity in Figures 4, 5 and 6. $f 1$ and $\mathrm{f} 2$ are frequency components (in $\mathrm{kHz}$ ). Figure 4 is the spectral peak of the block DFT filters detection, and Figures 5 and 6 are the spectral peak corresponding to the block LMS detection and the BFFTAA-MAE detection. From Figures 4 and 6, we can find that the block DFT filters detection algorithm and the block LMS detection algorithm will lose their detection capability under the low SNR condition, and the BFFTAA-MAE detection algorithm will keep better detection capability at the same SNR. Due to adapting the block-FFT adaptive filter algorithm and matrix accumulative algorithm to suppress noise, the BFFTAA-MAE detection algorithm can detect the weak signal more effectively from the background noise than the block DFT filters detection algorithm and the block LMS detection algorithm.

Then the performance of the detection algorithm is described in Figure 7 with the Receiver Operating Characteristics (ROC). The block DFT filters detection and the block LMS detection should have the proximate performance. There is bit error in Figure 7 between the block DFT filters detection and the block LMS detection mainly because of the simulation error. From Figure 7, the BFFTAA-MAE detection still has higher detection probability and lower false alarm probability at $-5.8 \mathrm{~dB}$

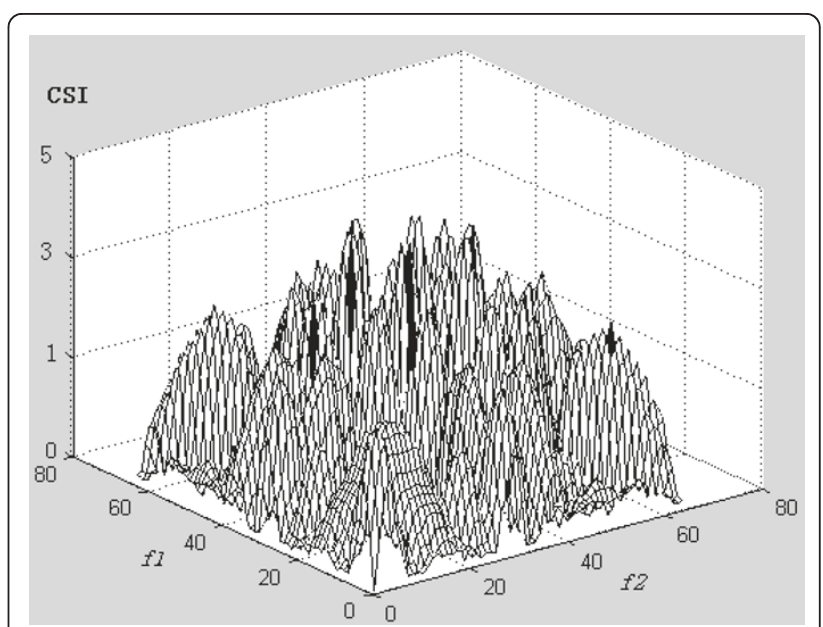

Figure 4 The spectral peak of the block DFT filters detection. 


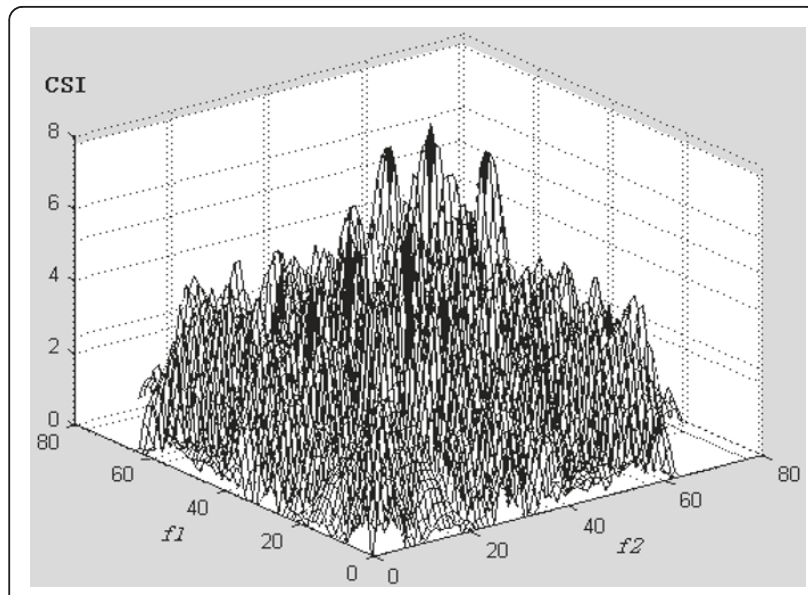

Figure $\mathbf{5}$ The spectral peak of the block LMS detection.

SNR if the detection probability $80 \%$ is taken as the performance standard. For uniting the advantage of the BFFTAA algorithm and matrix accumulation estimation, the BFFTAA-MAE algorithm has better performance than the block DFT filters and the block LMS detection. Obviously, the BFFTAA-MAE algorithm can degrade the strong noise to raise the weak signal step by step, and implement the weak signal detection from strong background noise. In comparison, the algorithm can better adapt to the complex radio environment and work in low SNR detection environment, and has advantageous over the performance of other two detection algorithms.

\section{Conclusion}

Since the cognitive radio can be used widely in communication, the spectrum cognitive algorithm will be given more and more importance. For the requirement of the cognitive radio, the new detection algorithm of the primary user's signal is introduced for the signal detection

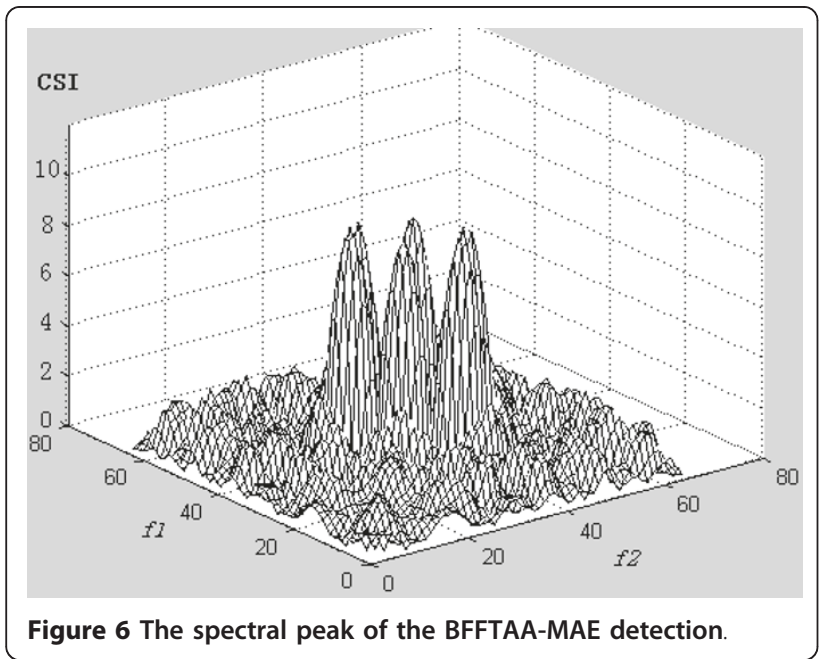

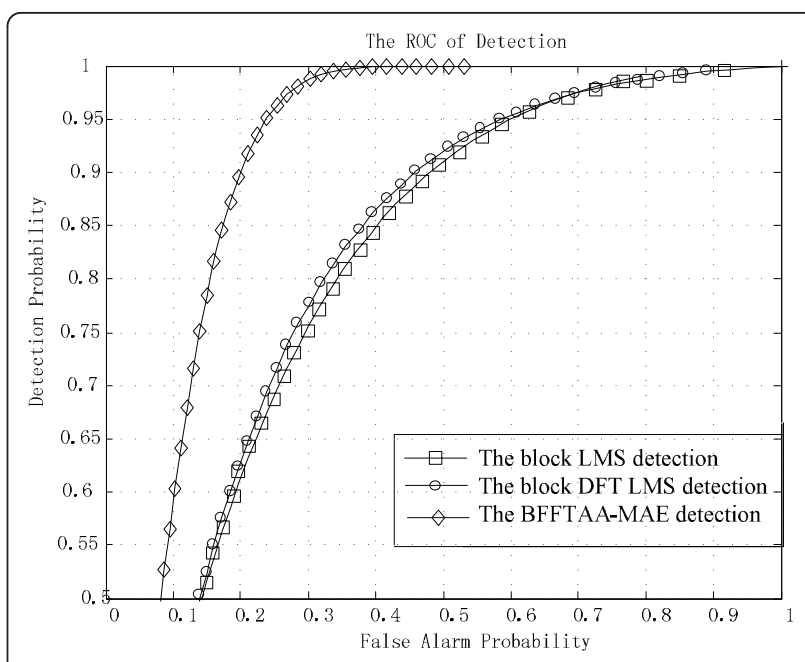

Figure 7 The ROC of the detection algorithm with SNR $=-5.8$ dB

from the background noise in the paper. Its core is to acquire the frequency-domain estimate value $\hat{S}$ through matrix accumulative calculation of the data processed by the Block-FFT adaptive filter algorithm. The result of the detection will be obtained by decision to the frequency-domain estimate value.

By simulation, it is obvious that the BFFTAA-MAE detection algorithm can solve the problem of the signal detection in low SNR, in other words, the algorithm greatly improves the detection performance. The detection algorithm can detect the primary user's signal, and collect spectrum-hole for cognitive users to communication. Because of adapting the block-FFT adaptive filter algorithm and matrix accumulative algorithm, the BFFTAAMAE detection algorithm can suppress not only the white noise but also colored noise. So the detection algorithm has definite prospect in the cognitive radio field.

\section{Competing interests}

The authors declare that they have no competing interests.

\section{Author details}

${ }^{1}$ National Mobile Communications Research Laboratory, Southeast University, Nanjing 210096, China ${ }^{2}$ Key Laboratory of Electronic Restriction, Hefei 230037, China

Received: 21 January 2011 Accepted: 1 September 2011 Published: 1 September 2011

\section{References}

1. A Sahai, Some fundamental limits on cognitive radio, in Proceedings of Allerton Conference, Monticello (2004)

2. Danijela Čabrić, Shridhar Mubaraq Mishra, Daniel Willkomm, Robert Brodersen, Adam Wolisz, Corvus: a cognitive radio approach for usage of virtual unlicensed spectrum. Berkeley Wireless Research Center White Paper (2004) 
3. J Lansford, Universal radio: making new spectrum, in Fourth Annual International Symposium On Advanced Radio Technologies, Boulder, CO (2002)

4. D Cabric, SM Mishra, RW Brodersen, Implementation issues in spectrum sensing for cognitive radio, in 38th Annual Asilomar Conference on Signals, System and Computers (2004)

5. S Soliman, Cognitive radio: key performance indicators. in QUALCOMM Incorporated (2004)

6. M Ghozz, M Dohler, F Marx, J Palicot, Cognitive radio: methods for the detection of free bands. Comptes Rendus Physique 7(7), 794-804 (2006) doi:10.1016/j.crhy.2006.07.009

7. K Jaiswal, Spectral sensing for cognitive radio: estimation of adaptive frequency hopping signal, in IEEE Region 10 Conference (TENCON 2008), Hyderabad, India, 2614-2618 (Nov 19-21, 2008)

8. R Tandra, A Sahai, SNR walls for signal detection. IEEE J Select Top Signal Process. 2(1), 4-17 (2008)

9. GA Clark, SK Mitra, SR Parker, Block implementation of digital filters. IEEE Trans Circuits Syst. 28(6), 584-592 (1981). doi:10.1109/TCS.1981.1085018

10. ER Ferrara, Fast implementation of LMS adaptive filters. IEEE Trans ASSP. 28(4), 474-475 (1980). doi:10.1109/TASSP.1980.1163432

11. B Farhang-Boroujeny, Adaptives Filters: Theory \& Applications (Wiley, UK), (1998)

12. C-C Hu, W-C Jhu, Adaptive block DFT-LMS signal detection in ultrawideband communications. in Proceedings of the 6th IEEE Conference on Consumer Communications and Networking Conference, Las Vegas, NV, USA 543-544 (2009)

13. B Atrouz, A Alimohad, B Alssa, An effective jammers cancellation by mean of a rectangular array antenna and a sequential block LMS algorithm: case of mobile sources. Prog Electromagn Res C. 7, 193-207 (2009)

14. H-J Zhou, B-H Sun, J-F Li, Q-Z Liu, Efficient optimization and realization of a shaped-beam planar array for very large array application. Prog Electromagn Res. 89, 1-10 (2009)

15. CL Nikias, Signal processing with high-order spectra. IEEE Signal Process Mag. 10(3), 10-37 (1993). doi:10.1109/79.221324

doi:10.1186/1687-6180-2011-51

Cite this article as: Wang et al:: Adaptive primary user's signal detection algorithm based on matrix accumulation estimation for cognitive radio. EURASIP Journal on Advances in Signal Processing 2011 2011:51.

\section{Submit your manuscript to a SpringerOpen ${ }^{\circ}$ journal and benefit from:}

- Convenient online submission

- Rigorous peer review

- Immediate publication on acceptance

- Open access: articles freely available online

- High visibility within the field

- Retaining the copyright to your article

Submit your next manuscript at $\gg$ springeropen.com 